\title{
Final word is near on dark-matter signal
}

\author{
An influential US experiment prepares to release \\ its first results.
}

\section{BY EUGENIE SAMUEL REICH}

$\mathrm{V}$ iewed end on, the arrays of photomultiplier tubes on the Large Underground Xenon (LUX) experiment look like beds of flowers. The hope is that they will capture sparks of light emitted when particles of dark matter collide with liquid xenon. With 122 detector tubes, LUX is much more sensitive than its closest rival in the competitive field of dark-matter searches - and in just days, physicists the world over will know whether that advantage has yielded definitive results.

The project, based at the Sanford Underground Research Facility in Lead, South Dakota, will release its first findings on 30 October. They are likely to reveal whether tentative dark-matter signals seen by other experiments are real, and will also inform ongoing discussions about how much more time and money should be spent on the hunt for dark matter. "The potential is there, and all the community is waiting with bated breath to see what they observe," says Juan Collar, a physicist who leads a rival experiment at the University of Chicago in Illinois.

Elena Aprile, a physicist at Columbia University in New York city who leads another competitor, XENON100, based at Gran Sasso National Laboratory near L'Aquila, Italy, is betting that LUX has not seen dark matter. "A null result is all that can be expected at this stage," she says. A LUX spokesman, physicist Daniel McKinsey of Yale University in New Haven, Connecticut, says simply: "We have a detector that is working very, very well."

LUX came online this year amid fierce debate. Scientists know from astronomical observations that five-sixths of the matter in the Universe is dark - making itself known mostly through its gravitational tug on bright matter - but attempts to detect it directly, on its presumed passage through Earth, have been fraught with controversy.

The DAMA/LIBRA experiment (Dark Matter Large Sodium Iodide Bulk for Rare Processes) at Gran Sasso reported a statistically significant signal more than 10 years ago, but physicists have not independently confirmed the result. In 2010, the Coherent Germanium Neutrino Technology experiment in Soudan, Minnesota, and the Cryogenic Dark Matter Search

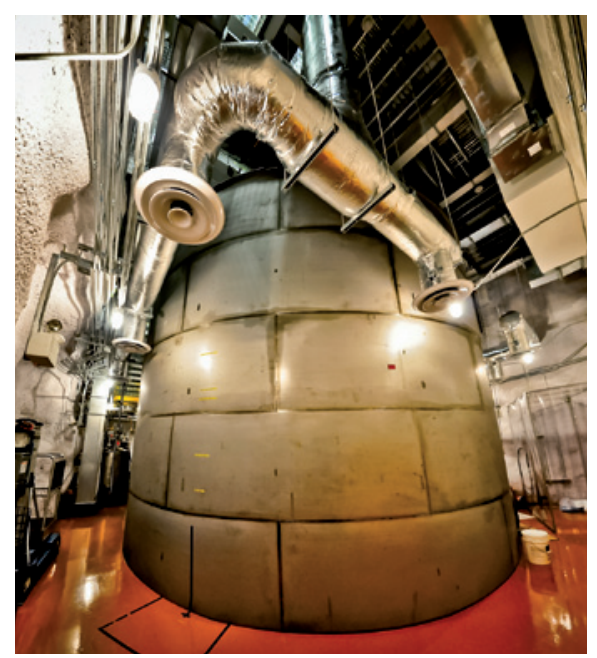

LUX could resolve a decade-long physics debate.

at the University of California, Berkeley, each reported tantalizing, but not statistically convincing, glimpses of potential dark matter; a year later, XENON100 saw no sign of the stuff. That prompted heated discussion over whether the experiment was sensitive to the lighter dark-matter particles that might have been glimpsed by the other two experiments.

Enter LUX, which will deliver its first results just as the US Department of Energy decides which of several dark-matter experiments should be given money to expand. LUX wants to install a larger, 7-tonne detector, in a proposed US\$30-million project called LUXZeplin. McKinsey argues that such experiments should be scaled up until they hit a physical limit - when the background noise from other weakly interacting particles becomes overwhelming. "That's a natural break point," agrees Jonathan Feng, a theoretical physicist at the University of California, Irvine.

One candidate for dark matter is the neutralino, a particle predicted by some supersymmetric theories of particle physics, in which particles are paired with heavier counterparts. If, as Feng expects, LUX sets a detection threshhold around three times more stringent than that of XENON100, it will rule out some types of neutralino. "There's an unbelievable amount of effort focused on the neutralino, so this upcoming announcement is quite important," he says. 